\title{
Relationship between geomagnetic classes' activity phases and their occurrence during the sunspot cycle
}

\author{
Frédéric Ouattara \\ Ecole Normale Supérieure, Université de Koudougou, Burkina Faso, Africa
}

\begin{abstract}
Four well known geomagnetic classes of activity such as quiet days activity, fluctuating activity, recurrent activity and shock activity time occurrences have been determined not only by using time profile of sunspot number Rz but also by using aa index values.

We show that recurrent wind stream activity and fluctuating activity occur in opposite phase and slow solar wind activity during minimum phase and shock activity at the maximum phase.

It emerges from this study that fluctuating activity precedes the sunspot cycle by $\pi / 2$ and the latter also precedes recurrent activity by $\pi / 2$. Thus in the majority the activities do not happen at random; the sunspot cycle starts with quiet days activity, continues with fluctuating activity and during its maximum phase arrives shock activity. The descending phase is characterized by the manifestation of recurrent wind stream activity.
\end{abstract}

Key words Classes of geomagnetic activity - Phases of geomagnetic classes - Sunspot cycle

\section{Introduction}

The long term aa and storm sudden commencement (SSC) data series (Mayaud, 1971; 1972; 1980) provide continuous information on geomagnetic activity from 1868 onwards. Using aa indices values and SSC data series from 1868 to 1978 (Legrand and Simon, 1989) classified geomagnetic activity into four classes. Recently Ouattara and Amory Mazaudier (2008) reported the percentage of their events for the period 1968-2005. Their work prolongs the previous work of Legrand and Simon (1989) from 1989 to 2005. Richardson et al. (2000) and Richardson and Cane (2002) have also classified geo-

Mailing address: Dr. Frédéric Ouattara, Ecole Normale Supérieure, BP 376 Burkina Faso; e-mail: ouattarafred@yahoo.fr; fojals@yahoo.fr magnetic activity into four classes by using aa values and in situ solar wind velocity. Ouattara and Amory Mazaudier (2008) showed that the conclusions obtained on the one hand by Legrand and Simon (1989) and on the other hand by Richardson and colleagues (2000) and Richardson and Cane (2002) are the same.

To determine geomagnetic classes of activity Legrand and Simon (1989) based their classification on 2 facts:

1) the contribution of shock wave to geomagnetic activity; this contribution is called shock activity.

2) the strong correlation between the aa index and the solar wind (Svalgaard, 1977). This correlation permits them to divide solar wind speed into three types: low speed, fluctuating and high speed. Each type generates one class of activity named respectively quiet activity, fluctuating activity and recurrent activity.

The criteria to determine the four classes of geomagnetic activity are given by Ouattara and Amory Mazaudier (2008) and must be expressed as: 
1) Quiet days activity is defined as the slow solar wind ( $\mathrm{v}<450 \mathrm{~km} / \mathrm{s})$ flowing continuously past the magnetosphere activity. Its concerns the days with daily aa $<20 \mathrm{nT}$.

The three other classes (shock activity, recurrent activity and fluctuating activity) constitute the disturbed geomagnetic activity classes and are obtained by taking into account the days with daily aa $>=20 \mathrm{nT}$. These classes are distinguished as follows:

2) Recurrent (stream) activity presents a continuing evolution during one solar rotation as well as during the following solar rotation. There is no SSC during the main phase. This class corresponds to high speed solar wind;

3) Shock activity is determined by the special storms which arise by random bursts and without a recurrence of 27 days during 2, 3 or 4 rotations.

4) Fluctuating activity is determined by subtracting from the disturbed activity (aa $>=20 \mathrm{nT}$ ) the shock activity and the recurrent activity.

The two last activities constitute the only transient activity among the four classes. Shock activity is related to very active spots on the solar disk and fluctuating activity is due to the existence of fluctuating solar wind jets.

It is important to underline that each class of activity determines solar wind structure and expresses solar magnetic field topology behaviour. Thus, physically different classes differ from others. Here, the key role is attributed to daily aa value for determining the class of activity not to the summarized daily aa values. The latter value is used to determine only the whole level of each class of activity (Ouattara and Amory Mazaudier, 2008).

In the following each geomagnetic class of activity will be expressed as: Quiet days activity (QA), Fluctuating activity (FA), Shock activity (SA) and Recurrent activity (RA).

To study the long-term variability of different solar classes of activity, we will use not only sunspot number $(\mathrm{Rz})$ which has been largely used to infer the long-term variability of the solar activity with annual averages available since 1700 (Eddy, 1976) but also the aa activity index computed by Mayaud (1971; 1972; 1973).

In this paper the long-term variability of the four classes of geomagnetic activity for the pe- riod 1868-2005 are compared each others in order to determine not only their time profiles which are well known (Legrand and Simon, 1989; Ouattara et al., 2008; Ouattara and Amory Mazaudier, 2008) but also to show their phases and to determine the relationship between them.

The present study is quite different from our work submitted to Journal des Sciences (Ouattara, 2008) where after separating the two components of solar magnetic fields by using aa values we try to find the relationship between each solar magnetic field component according to their time evolution and in term of their amplitude.

We show that solar magnetic field components are in opposite phase and the ratio between their amplitudes is one half.

The present paper starts by analyzing the relationship between geomagnetic activity index aa and sunspot number (Rz) and after determining geomagnetic classes activity phases and their occurrences in reference of $\mathrm{Rz}$ time profiles. A comparison among them will be also made.

\section{Data sets}

Sunspot number and aa geomagnetic indices values used in this study are provided by CETP (Centre d'Etude des phénomènes Terrestres et Planétaires) data base and NGDC (National Geophysical Data Center).

\section{Methodology}

Our methodology consists in indicating the process used to obtain the sum per year of aa values for each class of activity. The sum per year of aa values for each class of activity is obtained by adding the daily aa value per class of activity for a given year. This assumes that the daily class of activity is determined before adding the values.

For that, first we will indicate the method used to obtain the daily aa values for each class of activity and secondly we will show how to sum the values obtained. 


\subsection{Determination of each daily class of activity}

To determine the daily class of activity we organized the daily aa values by means of a pixel diagram. The pixel diagram is a colour diagram used to select the geomagnetic data as a function of the solar activity as described by solar rotation (27 days). This method was developed and used by Legrand and Simon (1989) for the determination of the four geomagnetic classes of activity for the period 1868-1978. This method was clearly exposed for the year 2003 and validated by Ouattara and Amory Mazaudier (2008): «Each pixel diagram has thirty one rows corresponding to the maximum day of the month. To obtain the thirty one rows the first four rows have been repeated at the end. The diagram is started by reading from the third row and finished at the 29th row. The corresponding day of the third row has mentioned on the left of the diagram and the corresponding year on the top of the diagram» (Ouattara and Amory Mazaudier, 2008).

In a pixel diagram the dates of SSCs are indicated to permit the determination of SA.

Each yearly pixel diagram is characterized by its colour repartition as a function of a daily aa values. The colour code repartition is expressed as: white colour for aa $<10 \mathrm{nT}$. This colour concerns a very quiet days periods; Blue colour for $10 \mathrm{nT}<=$ aa $<20 \mathrm{nT}$. All the white and blue colours characterize quiet days periods and correspond to aa $<20 \mathrm{nT}$.

The disturbed periods determined by aa $>=$ $20 \mathrm{nT}$ are characterized by the following colours according to the different values of aa. We have: green colour for $20<=$ aa $<30 \mathrm{nT}$; yellow colour for $30 \mathrm{nT}<$ aa $<=40 \mathrm{nT}$; orange color for $40 \mathrm{nT}<=$ aa $<60 \mathrm{nT}$; red colour for $60 \mathrm{nT}<=$ aa $<100 \mathrm{nT}$ and alive red for aa $>=100 \mathrm{nT}$.

For determining geomagnetic activity, pixel diagrams have been made for all values of aa since 1868, year after year until 2006. The continuum description of the geomagnetic activity as a function of solar activity is given by the picture.

For more details on the pixel diagram see Ouattara and Amory Mazaudier (2008).

The daily QA is determined by considering the days with aa $<20 \mathrm{nT}$. In a pixel diagram this determination is easy. One must choose the days with white colour (very quiet days: aa $<10$ $\mathrm{nT})$ and the days with blue colour $(10 \mathrm{nT}<=$ aa $<20 \mathrm{nT}$ ). The daily QA can be obtained without using the pixel diagram.

$\mathrm{RA}$ is due to the corotating solar wind streams. This class of activity is the class of recurrent (stream) activity from coronal holes: it presents a continuing evolution during one solar rotation as well as during the following rotation. There is no SSC during the main phase. This class corresponds to high speed solar wind.

In a pixel diagram this condition corresponds to $40 \mathrm{nT}<$ aa $<100 \mathrm{nT}$ with, $40 \mathrm{nT}<=$ aa $<60 \mathrm{nT}$ : orange colour and $60 \mathrm{nT}<=$ aa $<$ 100 nT: red colour.

It is important to note that to improve the determination of this class of activity by using in situ data measurements (solar wind parameters measured on board spacecraft) Ouattara and Amory Mazaudier (2008) added a supplementary condition determined by recurrent yellow colour in a pixel diagram and connected to aa by $30 \mathrm{nT}<=$ aa $<40 \mathrm{nT}$. According to these authors this supplementary condition corresponds to the class of associated corotating interactions regions activity.

SA corresponds to shock event. It is estimated in the pixel diagram by taking into account 2 or 3 disturbed days after no recurrence SSC date. To improve this class determination Ouattara and Amory Mazaudier (2008) show that this shock event is related to the interplanetary coronal mass ejections (ICMEs). Thus they added transient forward shocks generated ahead of fast ICMEs and related postshock flows determined in he pixel diagram by isolated aa $>40 \mathrm{nT}$ (orange, red and alive red colours) without SSC.

FA is expressed by the other daily values of aa $>=20 \mathrm{nT}$ which do not contribute to SA and RA.

\subsection{Determination of the sum per year of the level of each class of activity}

aa index can be expressed as: aa $=$ aa (quiet) + aa (disturbed) with aa (disturbed $)=$ aa $($ recurrent) + aa (shock) + aa (fluctuating). 
Each year activity is characterized by four components:

$\mathbf{a a}=\mathbf{a a q}+\mathbf{a a r}+\mathbf{a a s},+$ aaf where aaq $\Sigma$ aa (quiet)) is the sum of the daily aa indices for all the day with $a a<20 \mathrm{nT}$, aar is the sum of the daily aa indices of days with RA ( $\Sigma$ aa (recurrent)), aas is the sum of the daily aas indices of days with SA ( $\Sigma$ aa (shock)) and aaf is the sum of the daily aa indices of days with FA ( $\Sigma$ aa (fluctuating)).

It is important to note that there is another way of obtaining the sum of FA for a given year. This must be obtained by removing from the sum of the whole value of aa for a given year the sum of aa values which have contributed to QA, the sum of aa values of RA and the sum of aa values of SA.

This classification gives for each year a level of geomagnetic activity for the four classes of activity.

\section{Results and discussion}

Figure 1 gives the time evolution of $\mathrm{Rz}$ and the sum per year of geomagnetic activity i.e. the sum of aa during a year. It clearly appears from this figure what is well known: geomagnetic activity presents not only two peaks during sunspot cycle with the first peak which is related to sunspot activity and the second which is out phase but also 11-year periodicity as $\mathrm{Rz}$ (e.g. Echer et al., 2004; Hady, 2006). One must conclude that there is no correlation between $\mathrm{Rz}$ and geomagnetic activity expressed here by aa index.

Geomagnetic indices must be divided into three intervals which are (1) aa $<10 \mathrm{nT}$ for very quiet days activity, (2) aa $<20 \mathrm{nT}$ for QA and (3) aa $>=20 \mathrm{nT}$ for disturbed days activity.

The disturbed days activity to gather together FA, RA and SA (see Legrand, 1984, Legrand and Simon, 1989, Ouattara and Amory Mazaudier, 2008; Ouattara et al., 2008).

Figure 2 gives the activities of very quiet days and sunspot. One can see that both activities are in opposite phase.

By taking that into account that: 1) sunspot activity expresses toroidal magnetic activity, 2) a linear relationship exists between very quiet days activity and quiet days activity (Ouattara et al., 2008) and 3) quiet days activity really

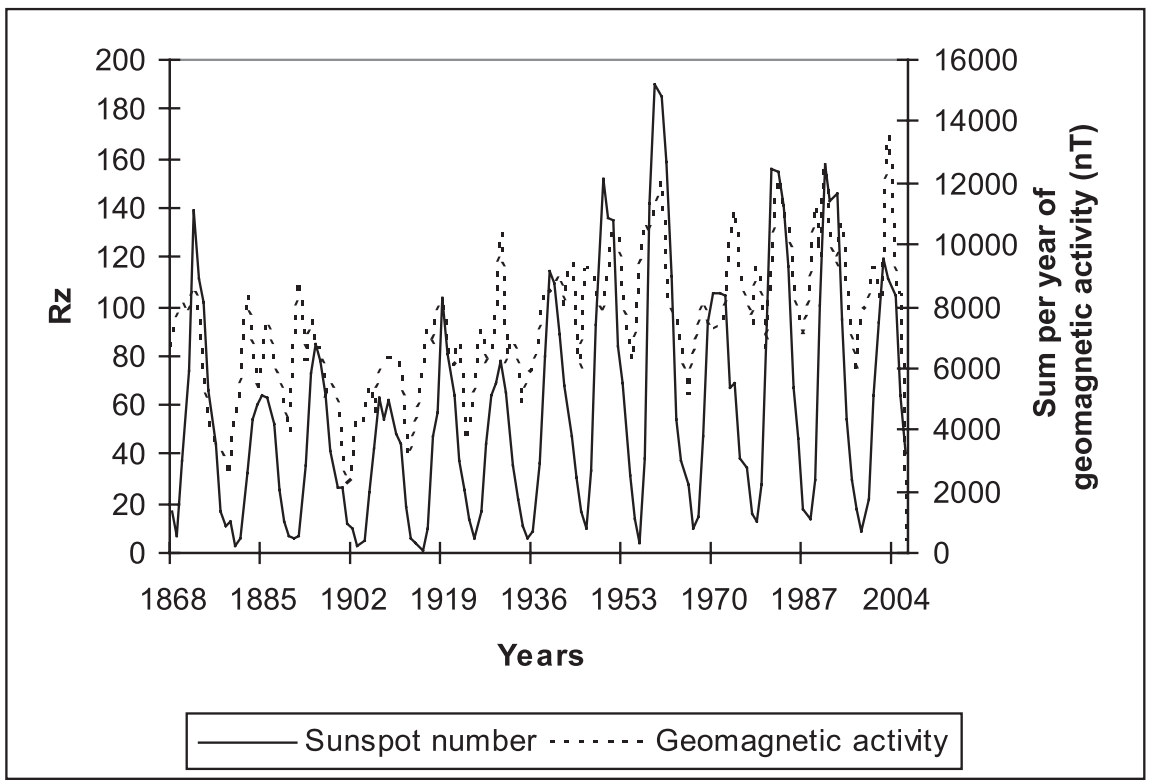

Fig. 1. Time profiles of $\mathrm{Rz}$ and sum per year of geomagnetic activity for the period 1868-2005. 


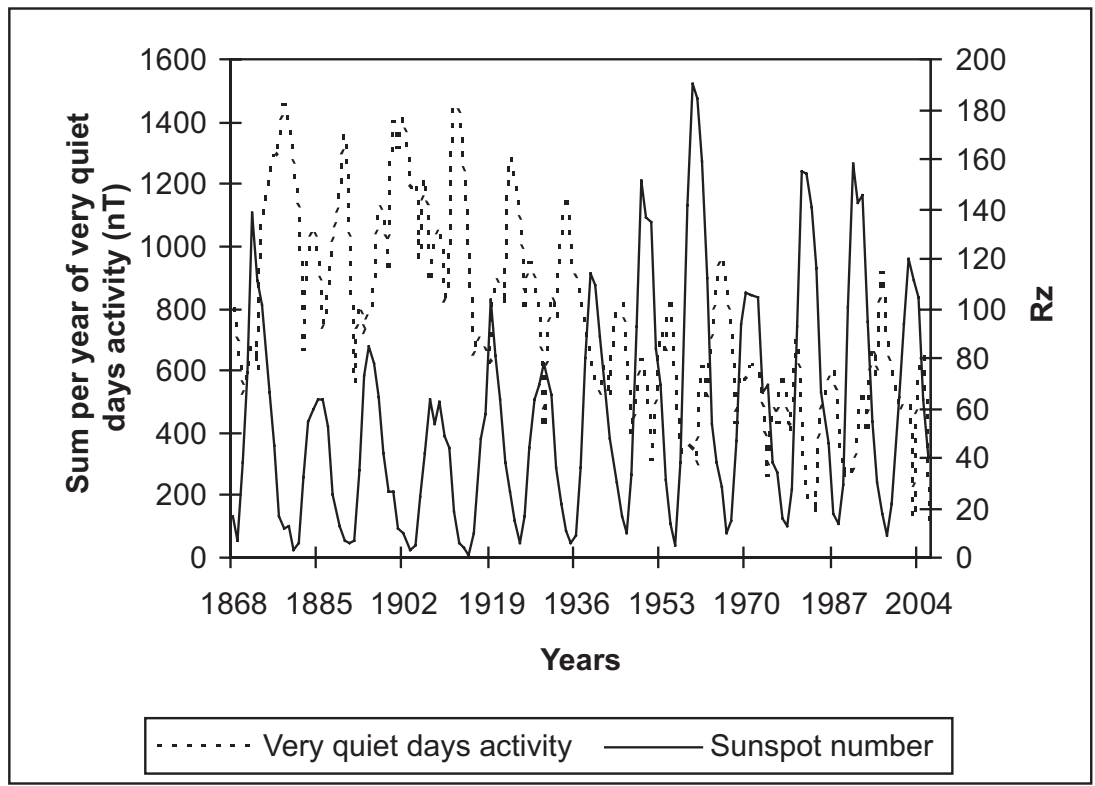

Fig. 2. Similar to fig. 1 but for Rz and Very quiet activity.

characterizes the signature of poloidal magnetic field activity (Legrand and Simon, 1989; Simon and Legrand, 1989), one must conclude that both fields are in opposite phase. This result has been also observed by other authors (Legrand and Simon, 1989; Luhmann et al., 2002; Ouattara, 2008). It also emerges from fig. 2 that the amplitudes of QA and Rz exhibit reverse development. Thus the year of greater (respectively smaller) $\mathrm{Rz}$ corresponds to the year of smaller (respectively greater) QA. Thus the knowledge of $\mathrm{Rz}$ amplitude infers a rough estimate of the amplitude of QA.

Figure 3 expresses the relationship between FA and sunspot activity. Minimums of sunspot arrive during the maximum of FA and the maximums of sunspot occur during the manifestation of the second peak or in the course of declining phase of FA. It can be concluded that FA precedes sunspot activity by $\pi / 2$. The second peak in FA graph expresses that some FA arrive at $\mathrm{Rz}$ maximum and consequently some geomagnetic activities with greater aa can contribute to FA.

It can be also seen that the amplitudes of $\mathrm{Rz}$ and FA develop together. Thus the greater (respectively smaller) $\mathrm{Rz}$ corresponds to the greater (respectively smaller) amplitude of FA. Thus when Rz amplitude is known it is possible to give a rough estimate of FA amplitude.

The comparative analysis of fig. 2 and fig. 3 shows on the one hand that greater (respectively smaller) FA corresponds to smaller (respectively greater) QA and on the other hand that through the solar cycle some intense FA with intense QA occur during Rz minimum.

This comparative analysis shows that QA occurs at Rz minimum QA and FA occurs during $\mathrm{Rz}$ ascending phase.

Figure 4 shows the time series of $\mathrm{Rz}$ and RA. It emerges that there is no RA during the maximums of $\mathrm{Rz}$ and the maximums of RA occur during the declining phases of Rz. This kind of evolution shows that Rz precedes RA by $\pi / 2$.

From fig. 4 one can see that during cycle 22 there is no RA. The addition of the absence of RA during Rz maximum phase to the above observation, allowed us to conclude that Rz cycle and RA cycle are not managed by the same solar phenomenon. 


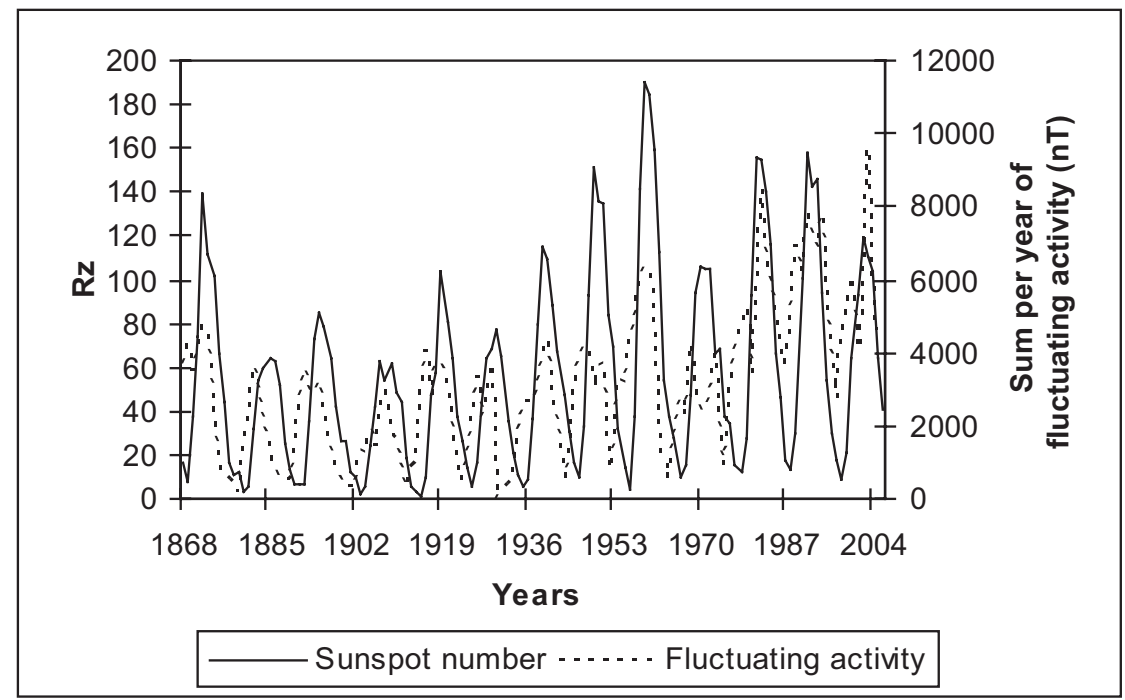

Fig. 3. Similar to fig. 1 but for Rz and Fluctuating activity.

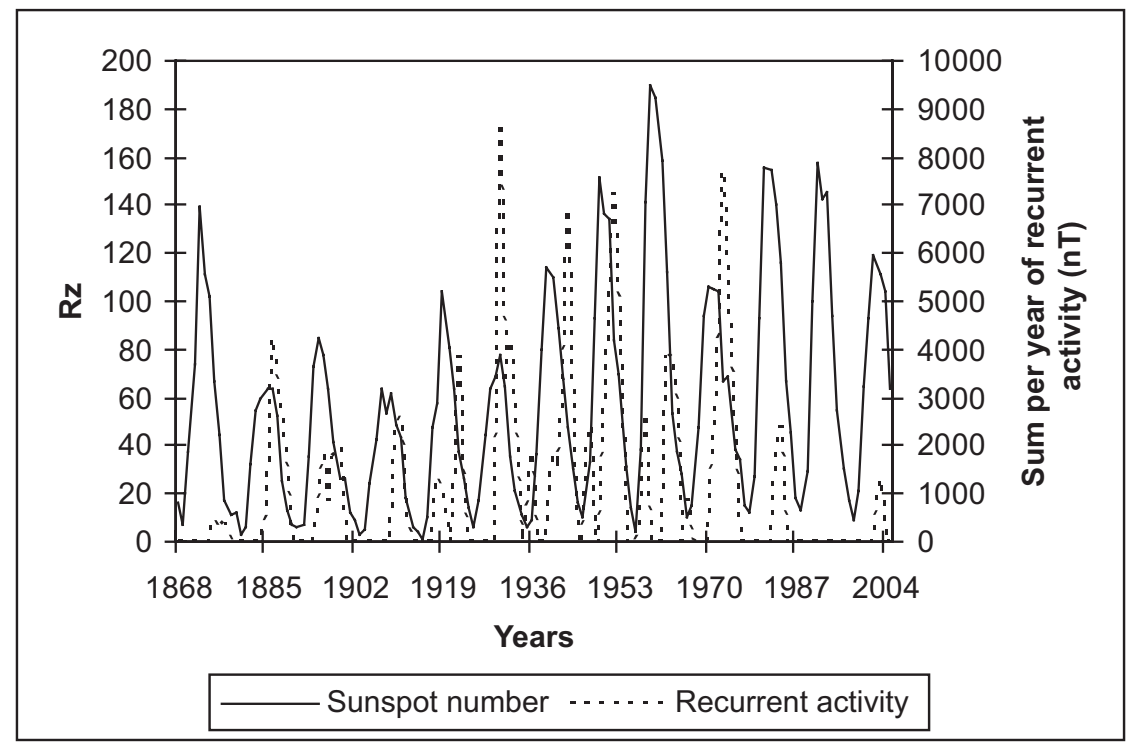

Fig. 4. Similar to fig. 1 but for Rz and Recurrent activity.

From the comparison between QA, FA and RA occurrences through the solar cycle it follows that RA happens during the declining phase while the formers occur respectively dur- ing minimum phase and increased phase.

Figure 5 shows the time series of SA and Rz. It can be seen that most SA comes at sunspot maximum. This indicates that SA and 


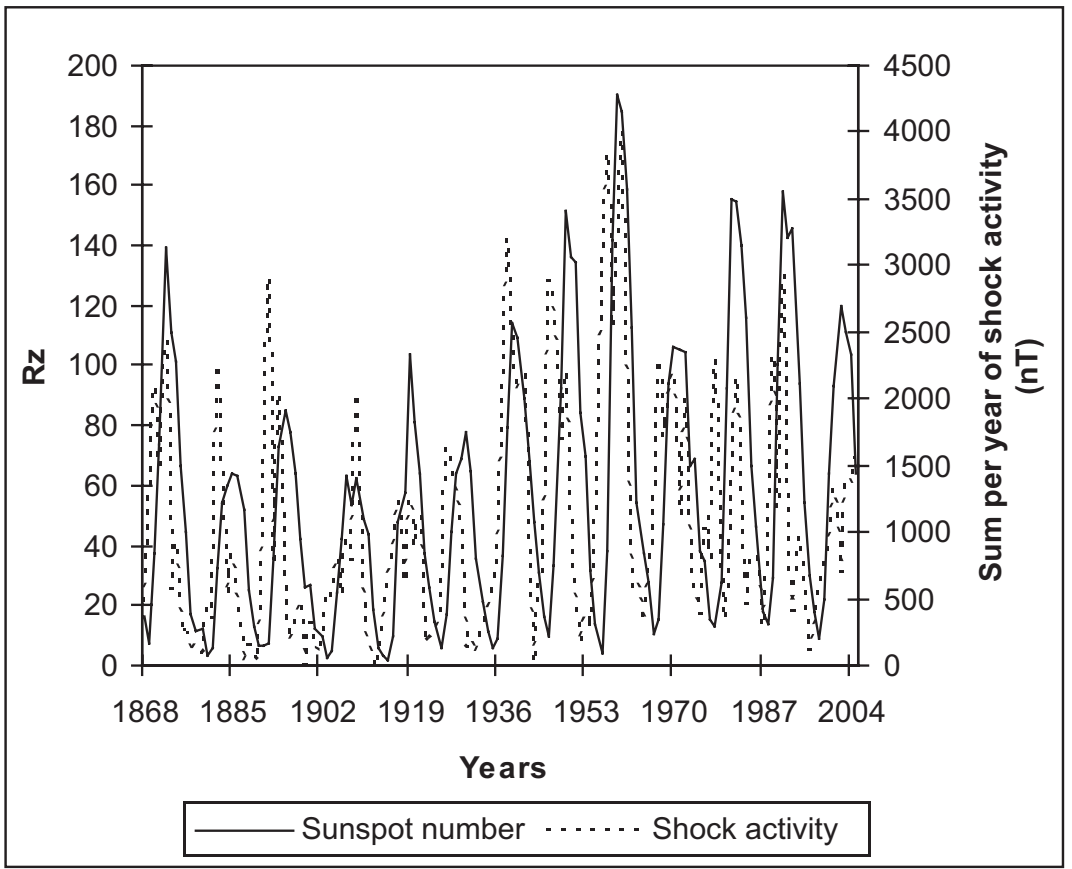

Fig. 5. Similar to fig. 1 but for Rz and Shock activity.

$\mathrm{Rz}$ are in phase and most $\mathrm{SA}$ is due to CMEs (Coronal mass ejections) as is well known.

Few SA are out of phase of $\mathrm{Rz}$ and precede it. This observation can be attributed to the error coming from the method of SA determination. Because of the occurrence of FA through the solar cycle (see fig. 4) and by reference to the classes activity determination method (see Ouattara and Amory Mazaudier, 2008) the few SA out of phase of $\mathrm{Rz}$ are the contribution of FA in SA.

Figure 5 shows that the major FA which contributed to SA occurred before the 20th Century. This highlights that the methods used for the determination of SA are more and more precise. As a consequence SA during the 20th Century they are exclusively due to CMEs.

When we plot the major activity due to aa $>$ $100 \mathrm{nT}$ and compare its activity with that of Rz (fig. 6) we confirm what has been previously said and can affirm that the major SA is produced by intense storms generated by CMEs.

The comparison between fig. 5 and fig. 6 shows that SA out of phase of $\mathrm{Rz}$ is not due to major activity. This observation permits us to conclude that SA is due to major storms during the last century. Such result points out the change in solar activity. This has been already pointed out by 1) Ouattara et al. (2008) in terms of decreasing of the number of quiet days, by 2) Rouillard et al. (2007) in terms of change in the dipole magnetic field and by 3) Svalgaard and Cliver (2007) in terms of the increase in solar wind speed. Ouattara and colleagues (2008) show that each term of change contributes to show the decreasing of the number of quiet days.

Figure 7 expresses the time evolution of RA and FA. We find that both activities are in opposite phase. The minimum of one arrives at the maximum of the other. This opposite phase was previously noted by Legrand and Simon (1989) and Ouattara (2008). This time evolution shows that through the solar cycle FA occurs during increased phase while RA happens during decreased phase. 


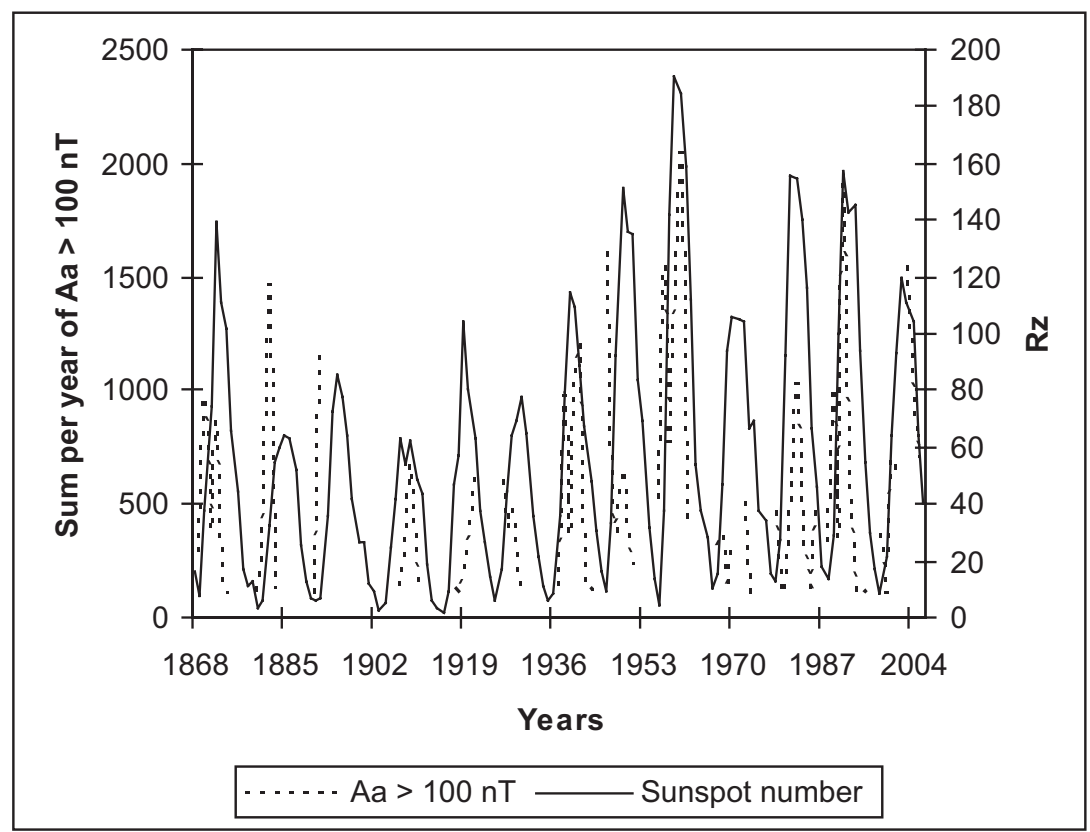

Fig. 6. Similar to fig. 1 but for $\mathrm{Rz}$ and aa $>100 \mathrm{nT}$.

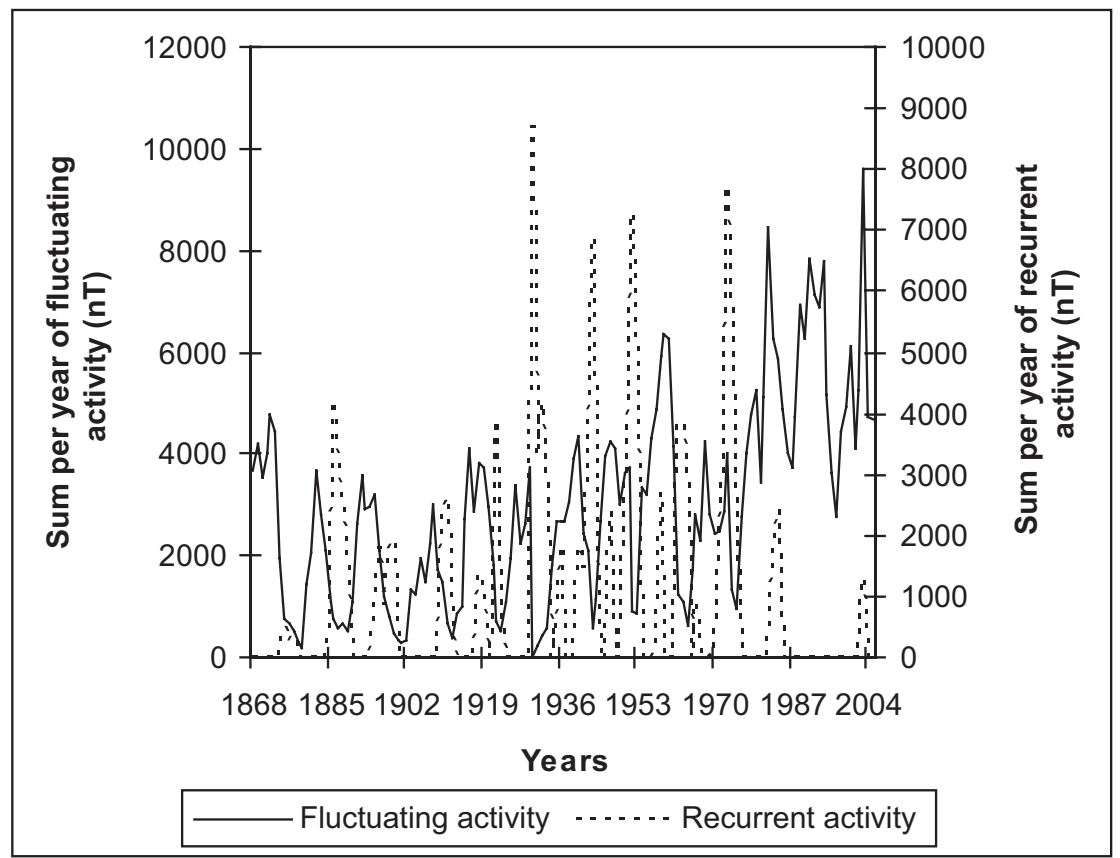

Fig. 7. Similar to fig. 1 but for Fluctuating activity and Recurrent activity. 
Summarizing, it emerges that the different time occurrences of the different activities permit us to indicate their position in the time profile of Rz. The minimum of $\mathrm{Rz}$ coincides with the manifestation of slow solar wind activity (QA) and during its ascending phase there are the events due to FA. In Rz maximum CMEs produce SA and when the declining arrives it is the manifestation of RA. The division of the different activities shown here was reported by Ouattara (2008) analysing the results shown by Legrand and Simon (1989) according to the relationship between the solar cycle and geomagnetic activity cycle.

The different time profiles of the four classes of geomagnetic activity let us conclude that the yearly frequencies of each activity differ and the contribution of each class of activity to the whole geomagnetic activity is also different and changes through time.

Ouattara and Amory Mazaudier (2008) show that from 1868 to 2004 geomagnetic activity level is constituted by $14 \%$ of SA, $14 \%$ of RA, $40 \%$ of FA and $32 \%$ of QA.

The yearly frequencies of each class have been given by Legrand and Simon (1989) for the period from 1868 to 1978 . Overthe time during this period yearly frequency occurrences are $8.5 \%$ for SA, $7 \%$ for RA, $17.5 \%$ for FA and $67 \%$ for QA.

\section{Conclusions}

The different classes of geomagnetic activity do not occur on average at random. Fluctuating activity produced by fluctuating solar wind stream precedes the sunspot cycle while the latter goes ahead on shock activity which is due to CMEs. Recurrent wind stream activity generated by high solar wind coming from coronal holes arrives after shock activity. Thus the sunspot cycle begins with slow solar wind activity continues with fluctuating wind stream activity and during its maximum arrive shock activity produced in majority by CMEs. The cycle finishes its evolution in descending phase by the manifestation of recurrent High solar wind speed coming from coronal holes.
Through the cycle the contribution of each class of activity to the whole geomagnetic activity differs from one class to another. The contribution of each class to geomagnetic activity is not constant in time. Moreover the yearly frequency occurrence of each class of activity also differs from one class to another class and per class, varies through the time.

\section{Acknowledgments}

We thank all data providers such as CETP (Centre d'Etude des phénomènes Terrestres et Planétaires) and NGDC (National Geophysical Data Center) for respectively aa data and Rz data.

We thank here Legrand Jean Pierre, Simon Paul and Christine Amory Mazaudier for their help and encouragement which have permitted us to understand solar phenomena and their link to terrestrial events. We also remember Simon Paul who died on January 20, 2008.

\section{REFERENCES}

Echer, E., W.D Gonzalez, A.L.C. Gonzales, A. Prestes, L.E.A. Vieira, A. DAl LAgo, F.L. Guarnieri and N.J. SCHUCH (2004): Long-term correlation between solar and geomagnetic activity, Journal of Atmospheric and Solar-terrestrial Physics, 66, 1019-1025.

EDDY, J.A. (1976): The maunder minimum, Science, 192, 1189-1202.

HADY, A.A. (2006): Solar activity and its effects during the last 23 cycles, Historical events and people in aeronomy, geomagnetism and solar-terrestrial physics, Band/volume VII, Heft $/ \mathrm{N}^{\circ} \mathrm{I}$.

LEGRAND, J.P. (1984): Introduction élémentaire à la physique cosmique et la physique des relations soleilterre, Territoire des terres australes et antarctiques françaises, pp. 306.

LEGRAND, J.P and P.A. Simon (1989): Solar cycle and geomagnetic activity: A review for geophysicists. Part I. The contributions to geomagnetic activity of shock waves and of the solar wind, Annales geophysicae, 7 (6), 565-578.

Luhmann, J.G., Y. Li., C.N. Arge, P.R. Gazis and R. UlRICH (2002): Solar cycle changes in coronal holes and space weather cycles, J. Geophys. Res., 107 (A8), doi: 10.21029/2001JA007550.

MaYAud, P.N. (1971): Une mesure planétaire d'activité magnétique basée sur deux observatoires antipodaux, Ann, géoph., 27, 71.

MAYAud, P.N. (1972): The aa indices: a 100-year series characterizing the magnetic activity, J. Geophys. Res., 77 (34), 6870-6874 
MAYAud, P.N. (1973): A hundred year series of geomagnetic data, 1868-1967, indices aa, Storm sudden commencements, IAGA Bull., 33, 252 (IUGG Publ. Office, Paris, Mayaud).

Mayaud, P.N. (1980): Derivation, Meaning, and Use of Geomagnetic Indices, Geophys. Monogr. Ser., vol. 22, AGU, Washington, D. C. Moos, N. A. F. (1910), Colaba Magnetic Data, 1846 to 1905, 2, The Phenomenon and its Discussion, 782 pp., Cent. Govt. Press, Bombay.

OuATtARA, F. (2008): Solar magnetic fields components: Phases, Profiles and their relationship, submitted to Journal des Sciences.

OuATtARA, F. and C. AMORY-MAZAudier (2008): Solar-geomagnetic activity and Aa indices toward a Standard, Journal of Atmospheric and Solar-Terrestrial Physics, doi:10.1016/j.jastp.2008.05.001

Ouattara, F., C. Amory-Mazaudier, M. Menvielle, P. SiMON and J-P. LEGRAND (2008): On the long term change in the geomagnetic activity during the XXth century, submitted in revised form to Annales Geophysicae.

Richardson, I.G., E.W. Cliver and H.V. Cane (2000): Sources of geomagnetic activity over the solar cycle: Relative importance of coronal mass ejections, high- speed streams, and slow solar wind, Journal of Geophysical Research, 105 (A8), 18200-18213, August 1.

Richardson, I.G. and H.V. CANE (2002): Sources of geomagnetic activity during nearly three solar cycles (1972-2000), Journal of Geophys. Res., 107 (A8), 1187.

Rouillard, A.P., M. LockWOOD and I. FinCH (2007): Centennial changes in the solar wind speed and in the open solar flux, J. Geophys. Res., 112, A05103, doi: 10.1029/2006JA012130.

Simon, P.A. and J. P. LEGRAND (1989): Solar cycle and geomagnetic activity: A review for geophysicists. Part II. The solar sources of geomagnetic activity and their links with sunspot cycle activity, Annales geophysicae, 7 (6), 579-594.

SvAlgaARD, L. (1977): Geomagnetic activity: dependence on solar wind parameters, in Coronal holes and high speed wind streams, edited by J.B. ZIRKER, (Colorado Ass. Univ. Press. Boulder), 371-432.

SvaAlgard, L. and E.W. Cliver (2007): Interhourly variability index of geomagnetic activity and its use in deriving the long-term variation of solar wind speed, J. Geophys. Res., 112, A10111, doi:10.1029/2007JA012437.

(received September 22, 2008; accepted November 12, 2008) 